\title{
Análise de fluxo multifásico em solo não saturado para verificação da vulnerabilidade ambiental de bacias de contenção de tanques de armazenamento de combustível
}

\author{
Multiphase flow analysis in unsaturated soils for evaluation of environmental \\ vulnerability of diversion ponds around oil storage tank areas
}

Vitor Aderaldo Demétrio de Souza', Marco Aurélio Holanda de Castro²

口

\begin{abstract}
RESUMO
O presente trabalho apresenta resultados de estudos analitico-experimentais visandoà verificação das condições deestanqueidade de bacias de contenção de tanques de armazenamento de combustíveis de um terminal portuário situado no Nordeste brasileiro. Com esse objetivo, foi efetuado estudo de caso com foco na avaliação da vulnerabilidade ambiental de duas áreas de estocagem distintas, para as quais foi realizada uma simulação transiente do avanço da frente de contaminação em um cenário hipotético de grande derramamento acidental de combustível em um terreno não saturado. Para o desenvolvimento dos estudos, procedeu-se à realização de uma extensa campanha de investigações geotécnicas de campo e de laboratório e à realização de simulações numéricas de fluxo multifásico, com o emprego do método dos elementos finitos.
\end{abstract}

Palavras-chave: solo não saturado; simulação numérica; fluxo multifásico; hidrocarboneto.

\begin{abstract}
This work presents results of analytical and experimental studies concerning the evaluation of permeability conditions of spill diversion ponds around fuel storage tanks located in a Brazilian northeast industrial plant. For this purpose, a case study was carried out in order to assess the environmental vulnerability of two distinct storage areas, by means of transient numerical simulations of contaminant advance in unsaturated soil after a hypothetical accident with hydrocarbon spill. For the development of the study, extensive geotechnical field and laboratory investigations were carried out, and numerical multiphase flow simulations were accomplished with the use of the finite element method.
\end{abstract}

Keywords: unsaturated soil; numerical simulation; multiphase flow; hydrocarbon.

\section{INTRODUÇÃO}

O estudo das condições de estanqueidade de bacias de contenção de tanques em áreas de armazenamento de combustíveis tem recebido grande impulso no Brasil nos últimos anos, em virtude de restrições normativas e de novas exigências de legislação.

Nesse contexto, a avaliação de uma possível contaminação do subsolo e do aquífero decorrente de um vazamento de hidrocarbonetos em cenário acidental passou a constituir importante foco de atenção de leis ambientais brasileiras.
Em âmbito nacional, a Lei no 9.966 (Lei do Óleo, de 28 de abril de 2000) estabelece que portos organizados, terminais, plataformas e equipamentos de apoio deverão dispor de estudo técnico para a definição das características das instalações e dos meios destinados ao recebimento e tratamento de resíduos e ao combate da poluição.

Quanto às exigências normativas vigentes, a NBR 17505-2, de 2006, estabelece que a área interna de bacias de contenção para tanques estacionários de líquidos inflamáveis deve ter condutividade hidráulica máxima de $10^{-6} \mathrm{~cm} \cdot \mathrm{s}^{-1}$, referenciada à água. Em casos em que as

$\square$

'Doutorando em Recursos Hídricos pela Universidade Federal do Ceará (UFC) - Fortaleza (CE), Brasil.

Engenheiro da Petrobras Transporte S.A. - Fortaleza (CE), Brasil.

2Doutor em Métodos Computacionais Aplicados à Engenharia pela Drexel University - Filadélfia (PA), Estados Unidos da América.

Professor Adjunto da UFC - Fortaleza (CE), Brasil.

Endereço para correspondência: Vitor Aderaldo Demétrio de Souza - Petrobras Transporte S.A - Avenida Santos Dumont, 2.122, sala 805 - 60150-161 - Fortaleza (CE), Brasil -

E-mail: vitor.ad.souza@gmail.com

Recebido: 29/03/10 - Aceito: 03/09/13 - Reg. ABES: 5310 
bacias de contenção tenham canaletas em concreto armado, com área de escoamento mínima de $900 \mathrm{~cm}^{2}$ (em torno dos tanques e demais pontos passíveis de vazamentos e direcionando, preferencialmente, os vazamentos para um sistema de drenagem), é admitida uma condutividade de $10^{-4} \mathrm{~cm} \cdot \mathrm{s}^{-1}$, também relativa à água.

Como métodos de impermeabilização admissíveis, a NBR 17505-2 recomenda, nos casos em que as condições locais sejam desfavoráveis, o revestimento dos diques com terra, aço, concreto ou alvenaria sólida.

Contudo, é conhecido o fato de que a eficiência das bacias de contenção não deve ser avaliada exclusivamente por critérios de condutividade em relação à água. Essa eficiência deve considerar, entre outras, as características dos produtos armazenados, a condutividade do material com relação ao produto armazenado, a profundidade do lençol freático e as distâncias das bacias de contenção dos pontos de exposição, tais como rios, lagos, estuários e áreas de proteção (CORSEUIL et al., 2006).

Internacionalmente, tal enfoque já consta em diversas legislações ambientais, e.g. U.S. Environmental Protection Agency (EPA, 2002) e California Codes (2003). A primeira referência, da Agência de Proteção Ambiental Americana, constitui a principal regulamentação relativa ao tema nos Estados Unidos (CORSEUIL et al., 2006). Em seu Registro Federal 40, CFR 112, Oil Pollution Prevention (2002), a U.S. EPA estabelece que as bacias de contenção, diques e bermas sejam "suficientemente impermeáveis" aos compostos armazenados nos tanques, sem especificar uma condutividade hidráulica máxima.

Nessa perspectiva, a U.S. EPA não simplesmente fixa um parâmetro hidráulico, mas exige que o material das bacias de contenção deva estar apto a conter derramamentos até que o poluente seja removido da bacia de contenção, considerando a especificidade local. Desse modo, um mesmo solo pode ser considerado "impermeável" a um determinado produto, ao mesmo tempo em que pode ser inadequado à contenção de outro combustível de propriedades diferentes (e.g., um fluido de menor viscosidade).

No Brasil, o Decreto Estadual (BA) n 11.235, de 2008, Art. 68, já incorpora essa última vertente ao preconizar que "toda a área de estocagem de produtos tóxicos, inflamáveis ou corrosivos deverá ser provida de dique de contenção devidamente impermeabilizado", sem fazer menção a valores admissíveis.

Tal abordagem requer a elaboração de investigações geotécnicas de campo e laboratório e estudos técnicos pormenorizados com vista a uma adequada caracterização do sítio a analisar.

Este trabalho tem como objetivo a apresentação de resultados obtidos em estudo de caso relativo à estanqueidade de bacias de contenção de tanques de armazenamento de combustíveis em operação e a avaliação de aspectos relevantes à inferência da vulnerabilidade ambiental do subsolo e do aquífero local em caso de grande derrame acidental de combustíveis. As simulações aqui apresentadas têm viés essencialmente prático e se restringiram à análise de fluxo multifásico em solo não saturado - não sendo abordados, no presente trabalho, aspectos associados ao transporte de contaminantes no meio poroso.

\section{METODOLOGIA}

A abordagem do problema adotada neste trabalho segue a tendência dos estudos mais recentes relacionados ao tema no Brasil (CORSEUIL et al., 2006; MACHADO, 2008), nos quais é estabelecido um tempo de resposta factível para o tratamento da ocorrência e estimada a profundidade de penetração do contaminante no solo. Uma bacia de contenção poderia ser, nessa ótica, considerada como suficientemente impermeável caso os eventuais impactos ambientais sejam compatíveis com o plano de contingência e as características locais.

Neste estudo, optou-se por não adotar limites de profundidade de contaminação pré-determinados, de modo que se permita que a decisão sobre a necessidade de se intervir em uma área específica fique a critério do analista (desde que essa decisão esteja provida de ferramentas de análise e de dados experimentais consistentes). Conforme sugerido por EPA (2002), ao proprietário ou operador de terminal deveria ser deixada a flexibilidade de decidir sobre o método a ser adotado na prevenção contra um eventual derramamento de óleo, devendo ser considerado suficiente qualquer método que satisfaça essa finalidade.

\section{Aspectos teóricos}

Com vista à realização dos estudos, foi empregado o software MOFAT (KATYAL et al., 1991, publicado pela U.S. EPA) para a análise de fluxo multifásico em meios porosos, desenvolvido com base no método dos elementos finitos. Com o uso dessa ferramenta, procedeu-se à análise do regime de fluxo em solos não saturados a partir do escalonamento da função de condutividade hidráulica e da curva característica de sucção dos solos (determinadas para o par água/ar), transformando-as em curvas multifásicas.

No caso em análise, a curva característica e a função de condutividade do solo são modificadas em função de suas tensões superficiais e interfacial, considerando-se os poros preenchidos com mais de uma fase líquida - o hidrocarboneto ou NAPL (Non Aqueous Phase Liquid). O modelo empregado utiliza as Equações 1 a 3:

$$
\begin{aligned}
& \phi \frac{\partial S_{W}}{\partial t}=\frac{\partial}{\partial x}\left[K_{W}\left(\frac{\partial h_{W}}{\partial x}+\rho_{R W} u_{Z}\right)\right]+\frac{\partial}{\partial z}\left[K_{W}\left(\frac{\partial h_{W}}{\partial z} \rho_{R W} u_{Z}\right)\right]+\frac{R_{W}}{\rho_{W}} \\
& \phi \frac{\partial S_{O}}{\partial t}=\frac{\partial}{\partial x}\left[K_{O}\left(\frac{\partial h_{O}}{\partial x}+\rho_{R O} u_{Z}\right)\right]+\frac{\partial}{\partial z}\left[K_{O}\left(\frac{\partial h_{O}}{\partial z} \rho_{R O} u_{Z}\right)\right]+\frac{R_{O}}{\rho_{O}} \\
& \phi \frac{\partial \rho_{A} S_{A}}{\partial t}=\frac{\partial}{\partial x}\left[\rho_{A} K_{A}\left(\frac{\partial h_{A}}{\partial x}+\rho_{R A} u_{Z}\right)\right]+\frac{\partial}{\partial z}\left[\rho_{A} K_{A}\left(\frac{\partial h_{A}}{\partial z} \rho_{R A} u_{Z}\right)\right]+R_{A}
\end{aligned}
$$


nas quais,

$\phi$ : porosidade - adimensional [-];

$S_{W}, S_{O}, S_{A}$ : saturação das fases água, óleo e ar, respectivamente [-]; $t$ : tempo $[\mathrm{T}]$;

$K_{W^{\circ}} K_{O^{\prime}} K_{A}$ : tensor de condutividade para as fases água, óleo e ar, respectivamente $[\mathrm{L} / \mathrm{T}]$;

$h_{W^{p}} h_{O^{\prime}} h_{A}$ : altura de água equivalente à carga de pressão da fase $\mathrm{p}$ - água

(w), óleo (o) ou ar (a) -, dada por $h_{P}=P_{P} / g \rho_{w}[\mathrm{~L}]$;

$P_{P}$ : pressão relativa à fase $\mathrm{p}\left[\mathrm{M} / \mathrm{LT}^{2}\right]$;

$g$ : aceleração da gravidade $\left[\mathrm{L} / \mathrm{T}^{2}\right]$;

$\rho_{W^{*}} \rho_{O^{\prime}} \rho_{A}$ : densidade das fases água, óleo e ar, respectivamente $\left[\mathrm{M} / \mathrm{LT}^{2}\right]$;

$R_{\omega^{\circ}} R_{O}, R_{A}$ : razão de transferência de massa por unidade de volume nas fases água, óleo e ar $\left[\mathrm{M} / \mathrm{L}^{3}\right]$;

$u_{Z}$ : vetor unitário gravitacional, no qual $z$ é a elevação - adotando-se sentido positivo para cima [-].

As relações de condutividade entre fases, saturações e pressões são descritas por meio da extensão para três fases do modelo de Van Genuchten, do qual derivam as cargas de pressão capilar definidas nas Equações 4 a 6:

$h_{A W}=h_{A}-h_{W}[\mathrm{~L}]$

$h_{O W}=h_{O}-h_{W}[\mathrm{~L}]$

$h_{A O}=h_{A}-h_{O}[\mathrm{~L}]$

Antes da entrada de óleo em um determinado ponto da massa de solo, o domínio é constituído por um sistema bifásico (ar e água) representado pela formulação de Van Genuchten (1980), conforme Equação 7:

$\bar{S}_{W}=\left[1+\left(\alpha h_{A W}\right)^{n}\right]^{m}$

na qual,

$\bar{S}_{W}$ é a saturação de água efetiva, definida pela Equação 8:

$\bar{S}_{W}=\frac{S_{W}-S_{M}}{1-S_{M}}$

$S_{M}$ é a saturação de água residual;

$\alpha\left[L^{-1}\right]$ e $n[-]$ são parâmetros de Van Genuchten para o meio poroso, sendo $m=1-\frac{1}{n}[-]$.

Após a entrada do óleo no meio poroso, o sistema passa a ser descrito com base nas Equações 9 e 10:

$\overline{\bar{S}}_{W}=\left[1+\left(\alpha \beta_{O W} \cdot h_{O W}\right)^{n}\right]^{-m}$
$\bar{S}_{T}=\left[1+\left(\alpha \beta_{A O} \cdot h_{A O}\right)^{n}\right]^{-m}$

na qual,

$\overline{\bar{S}}_{W}$ : saturação aparente de água [-];

$\bar{S}_{T}$ : saturação total líquida efetiva [-];

$\beta_{A O}$ : fator de escala estimado pela razão entre as tensões superficiais da água e do óleo [-];

$\beta_{O W}$ : fator de escala estimado pela razão entre a tensão superficial da água e a tensão interfacial água-óleo [-];

A saturação aparente da água, $\bar{S}_{W}$, é expressa pela Equação 11:

$\overline{\bar{S}}_{W}=\bar{S}_{W}+\bar{S}_{\text {OT }}$

na qual,

$\bar{S}_{O T}$ é a saturação efetiva de óleo no meio poroso [-], estimada pela equação de Land:

$\bar{S}_{O T}=\left[\frac{1-\bar{S}_{W}^{\min }}{1+R_{O W}\left(1-\bar{S}_{W}^{\min }\right)}-\frac{1-\overline{\bar{S}}_{W}^{\min }}{1+R_{O W}\left(1-\bar{S}_{W}^{\min }\right)}\right]$, se $\bar{S}_{W}>\bar{S}_{W}^{\min } ;$

$\bar{S}_{O T}=0$, se $\overline{\bar{S}}_{W} \leq \bar{S}_{W}^{\min }, \operatorname{com} R_{O W}=\frac{1}{\bar{S}_{R O}^{\max }}-1 \mathrm{e}$

$\bar{S}_{W}^{\text {min }}$ : mínima saturação efetiva de água [-], desde a entrada do NAPL no meio;

$\bar{S}_{R O}^{\text {max }}$ : máxima saturação efetiva residual de óleo [-], expressa por $S_{O} /\left(1-S_{M}\right)$. Os valores de saturação de água e óleo são determinados com base nas Equações 13 e 14:

$S_{W}=\left(1-S_{M}\right) \cdot\left(\overline{\bar{S}}_{W}-\bar{S}_{\text {OT }}\right)+S_{M}$

$S_{O}=\left(1-S_{M}\right) \cdot \bar{S}_{T}+S_{M}-S_{W}$

Os valores de condutividade $[\mathrm{L} / \mathrm{T}]$ das três fases são dados pelas Equações 15 a 17:

$K_{W i j}=k_{R W} \cdot K_{S W i j}$

$K_{O i j}=k_{R O} \cdot K_{S W_{i j}} / \eta_{R O}$

$K_{A i j}=k_{R A} \cdot K_{S W i j} / \eta_{R A}$

na qual,

$K_{R W^{\circ}} K_{R O^{\prime}} K_{R A}$ : condutividade relativa das fases água, óleo e ar [-]; 
$\eta_{R O}, \eta_{R A}:$ razão entre as viscosidades do óleo e do ar com relação à água, respectivamente [-];

$K_{S W_{i j}}$ : tensor de condutividade saturada da água $[\mathrm{L} / \mathrm{T}]$;

As expressões de condutividade relativa entre fases são apresentadas pelas Equações 18 a 20:

$K_{R W}=\bar{S}_{W}^{1 / 2} \cdot\left[1-\left(1-\bar{S}_{W}^{1 / m}\right)^{m}\right]^{2}$

$K_{R O}=\left(\bar{S}_{T}-\overline{\bar{S}}_{W}\right)^{1 / 2} \cdot\left[\left(1-\bar{S}_{W}^{1 / m}\right)^{m}-\left(1-\bar{S}_{T}^{1 / m}\right)^{m}\right]^{2}$

$K_{R A}=\left(1-\bar{S}_{T}\right)^{1 / 2} \cdot\left(1-\bar{S}_{T}^{1 / m}\right)^{2 m}$

O software empregado nas análises aplica o método de Galerkin para a solução numérica das equações diferenciais supracitadas com o uso de elementos finitos quadrangulares.

Um aspecto importante a ser mencionado diz respeito aos erros que podem ser cometidos na avaliação do avanço da frente de contaminação com o emprego da condutividade hidráulica do hidrocarboneto determinada a partir de relações entre densidade e viscosidade (formulação de Nutting), definidas pela Equação 21:

$K=\frac{k \cdot \rho \cdot g}{\eta}$

na qual,

$K$ : condutividade hidráulica saturada do fluido $[\mathrm{L} / \mathrm{T}]$;

$k$ : permeabilidade intrínseca do meio poroso $\left[\mathrm{L}^{2}\right]$;

$\rho$ : densidade do fluido $\left[\mathrm{M} / \mathrm{L}^{3}\right]$;

$g$ : aceleração da gravidade $\left[\mathrm{M} / \mathrm{T}^{2}\right]$;

$\eta$ : viscosidade dinâmica [M/LT].

Com base na formulação acima, a condutividade hidráulica saturada do solo ao produto armazenado poderia ser obtida a partir das propriedades dos fluidos de interesse, normalmente a água (w) e o produto $(\mathrm{p})$ :

$K_{P}=K_{W} \cdot \frac{\rho_{P}}{\rho_{W}} \cdot \frac{\eta_{W}}{\eta_{P}}$

De acordo com Machado (2008), essa relação conduz a resultados satisfatórios para meios granulares e inertes (e.g., areias), já que a permeabilidade intrínseca, nesses casos, pode ser de fato considerada uma propriedade exclusiva do meio poroso.

Todavia, para solos argilosos são frequentemente observadas diferenças significativas entre valores de condutividade medidos e calculados $\left(\mathrm{K}_{\mathrm{p}}\right.$ determinado a partir de $\left.\mathrm{K}_{\mathrm{W}}\right)$. Tais erros estariam associados a inúmeros fatores, tais como alterações na permeabilidade intrínseca do solo (pelo efeito de forças de atração elétrica entre o produto e a partícula argilosa, entre outros), interações físico-químicas e a diferença de polaridade entre a água e os líquidos orgânicos (representada pelos valores da constante dielétrica dos fluidos em análise).

De um modo geral, os valores de $\mathrm{K}_{\mathrm{p}}$ calculados para o hidrocarboneto a partir de $\mathrm{K}_{\mathrm{W}}$ tendem a ser inferiores aos medidos em laboratório, embora apresentem grande dispersão de resultados, inclusive exibindo valores superiores aos determinados experimentalmente.

Por outro lado, quando estimados a partir de valores de condutividade medidos por intermédio de outro hidrocarboneto, de constante dielétrica similar, os valores calculados de $\mathrm{K}_{\mathrm{P}}$ tendem a se aproximar dos valores determinados em laboratório (MACHADO, 2008).

\section{Características da área}

Para a realização das análises, foram selecionadas duas áreas de armazenamento de combustíveis de um mesmo terminal, mas com características geotécnicas bastante distintas: Área 1, local de armazenamento de óleo diesel, com solo de fundação silto-argiloso, e nível freático (NA) situado em profundidade de cerca de $5 \mathrm{~m}$; Área 2, local de armazenamento de óleo combustível, com solo superficial argilo-siltoso e NA situado a cerca de $2 \mathrm{~m}$ de profundidade.

Ambas as áreas estão em operação há vários anos e contêm tanques de combustíveis cercados de diques de contenção com solo compactado (com altura de $3 \mathrm{~m}$, aproximadamente). A superfície do terreno natural (TN) no interior das bacias encontra-se não impermeabilizada e não revestida (Figura 1).

\section{Dados experimentais}

As áreas selecionadas para este estudo receberam investigações geotécnicas de campo e de laboratório realizadas pelo Laboratório de Geotecnia Ambiental da Escola Politécnica da Universidade Federal da Bahia (MACHADO, 2008). As investigações abrangeram sondagens a trado, poços de inspeção, ensaios de permeabilidade de campo (Guelph), caracterização, permeabilidade de laboratório (água e diesel) e determinação de curvas características de sucção. Um resumo dos resultados obtidos, representativos dos solos das bacias do terminal em estudo, é apresentado a seguir.

As Tabelas 1 e 2 exibem os resultados dos ensaios de permeabilidade de campo e laboratório realizados em amostras do solo predominante nas áreas em estudo. Nessas tabelas, pode ser observado que os valores de condutividade hidráulica de campo e de laboratório para a água se mostraram relativamente próximos e que a condutividade ao óleo diesel excedeu, nos solos analisados, a referenciada à água, com $K_{O L E O} / K_{W}$ oscilando entre 4 e 668 , 


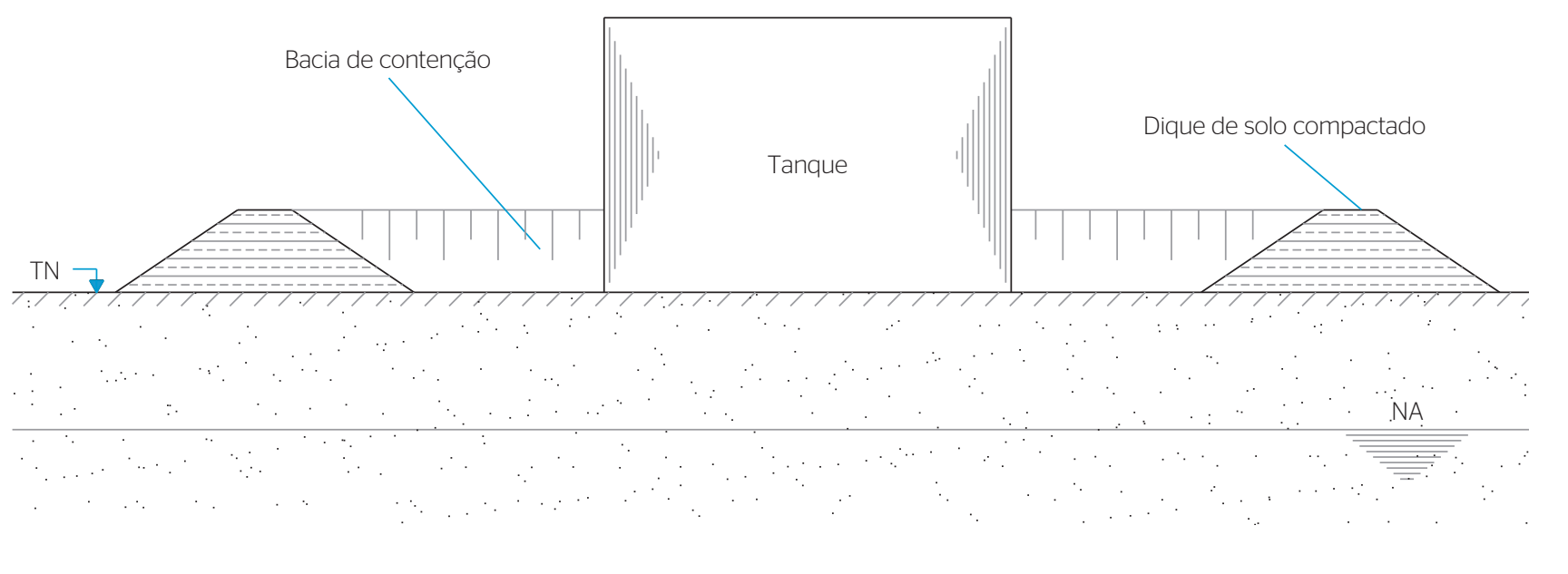

Figura 1 - Desenho esquemático das bacias de contenção avaliadas.

Tabela 1 - Condutividade hidráulica medida em campo para os solos das áreas 1 e 2.

\begin{tabular}{|c|c|c|c|}
\hline \multicolumn{2}{|c|}{ Área 1 - Argila siltosa } & \multicolumn{2}{|c|}{ Área 2 - Areia silto-argilosa } \\
\hline 0,50 & $<8,87 \mathrm{E}-08$ & 0,20 & $<7,75 \mathrm{E}-\mathrm{O} 8$ \\
\hline 0,20 & $<4,79 \mathrm{E}-08$ & 0,45 & $<6,39$ E-08 \\
\hline 0,40 & $<2,96 \mathrm{E}-08$ & 0,20 & 4,72E-05 \\
\hline 0,35 & 1,06E-05 & 0,20 & 8,28E-03 \\
\hline 0,70 & 1,73E-O7 & 0,20 & 6,83E-06 \\
\hline 0,15 & $<4,79 \mathrm{E}-08$ & 0,25 & 4,78E-05 \\
\hline 0,36 & 1,69E-O7 & 0,50 & 9,78E-08 \\
\hline- & - & 0,45 & 1,55E-06 \\
\hline- & - & 0,20 & 4,59E-07 \\
\hline- & - & 0,45 & $<1,54 \mathrm{E}-08$ \\
\hline- & - & 0,50 & $<1,05 \mathrm{E}-\mathrm{O} 7$ \\
\hline \multicolumn{4}{|c|}{ Valores médios (cm.s.1) } \\
\hline \multicolumn{2}{|c|}{ 1,97E-07 } & \multicolumn{2}{|c|}{ 2,72E-06 } \\
\hline
\end{tabular}

aproximadamente. Tais resultados, como era de ser esperado, contrariam a previsão efetuada a partir da equação de Nutting, na qual o valor de $K_{O L E O}$ deveria ser inferior ao de $K_{W}$ e equivaler, nesse caso, a cerca de $22 \%$ do último.

Comparando-se os resultados de permeabilidade de laboratório de ambas as áreas (Tabela 2) com o valor de $K_{W}$ preconizado na NBR 17505-2 $\left(10^{-6} \mathrm{~cm} \cdot \mathrm{s}^{-1}\right)$ para bacias sem sistema de drenagem, observa-se que somente a Área 1 atenderia ao referido critério. Nessa ótica, a simples aplicação do disposto na referência normativa citada exigiria a execução de obras de impermeabilização em somente uma das áreas em estudo (na Área 2).

Uma compilação de 108 resultados de laboratório atualmente disponíveis para diversas áreas de armazenamento em operação exibiu 
Tabela 2 - Condutividades determinadas em laboratório para os solos das Áreas 1 e 2.

\begin{tabular}{|c|c|c|c|}
\hline \multicolumn{2}{|c|}{ Área 1 - Argila siltosa } & \multicolumn{2}{|c|}{ Área 2 - Areia silto-argilosa } \\
\hline Água & Óleo diesel & Água & Óleo diesel \\
\hline 3,65E-07 & 6,67E-05 & 1,93E-06 & 7,25E-06 \\
\hline 3,88E-07 & 1,28E-04 & 5,56E-06 & 9,40E-05 \\
\hline- & 3,91E-05 & 3,43E-06 & 8,33E-05 \\
\hline- & 2,97E-04 & 1,81E-05 & - \\
\hline 4,50E-08 & 4,00E-06 & 9,93E-07 & - \\
\hline 1,10E-08 & 7,35E-06 & - & - \\
\hline- & 4,67E-06 & - & - \\
\hline - & 1,33E-O5 & - & - \\
\hline \multicolumn{4}{|c|}{ Valores médios (cm.s.1) } \\
\hline $2,02 \mathrm{E}-07$ & 7,00E-05 & $6,01 \mathrm{E}-06$ & $6,15 \mathrm{E}-05$ \\
\hline
\end{tabular}

valores medidos superiores aos calculados com base na equação Nutting em $71 \%$ dos casos, com valor médio de $K_{M} / K_{C}$ (razão entre as permeabilidades medida e calculada) igual a 231,6 , aproximadamente, com $76 \%$ dos valores inferiores a 100 e cerca de $84 \%$ inferiores a 200 (Figuras 2 e 3). Tais resultados provieram de ensaios de condutividade hidráulica realizados em solos de diversas áreas (permeados com diferentes produtos), em campanhas de investigação realizadas pelas Universidades Federais de Santa Catarina (UFSC) e da Bahia (UFBA), conforme descrito em Corseuil et al. (2006) e Machado (2008). Uma análise detalhada acerca de fatores intervenientes nos valores de condutividade de solos com referência a hidrocarbonetos foi realizada por Oliveira (2001).

$\mathrm{Na}$ Figura 4, são apresentadas as curvas características de sucção à água representativas das Áreas 1 e 2.

\section{Parâmetros da análise e condições de contorno}

Para a realização da simulação numérica do vazamento acidental de grande volume de combustível no interior das bacias de contenção, foram adotados os parâmetros relacionados na Tabela 3, empregando-se a mesma simbologia constante nas equações anteriormente apresentadas.

Para cada área analisada, foram empregados dois valores diferentes de condutividade do solo ao hidrocarboneto:

1. Valor de $K_{P}$ calculado a partir de $K_{W}$;

2. Valor de $K_{p}$ medido ou corrigido com base em resultados de ensaios de permeabilidade do solo ao óleo diesel.

Neste último caso - face às diferenças frequentemente observadas entre condutividades de solos medidas em laboratório, com hidrocarbonetos, e as estimadas a partir da permeabilidade intrínseca - , o valor de $K_{p}$ calculado foi multiplicado por um fator de correção representativo da divergência observada entre a permeabilidade medida com óleo diesel e a estimada a partir de $K_{W}$ para o hidrocarboneto em estudo (Tabela 4). A introdução do referido fator de correção baseia-se na observação de que os valores calculados de $K_{P}$ tendem a se aproximar dos valores determinados em laboratório quando estimados a partir de valores de condutividade medidos para outro hidrocarboneto, de constante dielétrica similar (MACHADO, 2008).

Como condição de contorno associada ao problema, foi adotada carga de pressão de óleo constante e igual a $3 \mathrm{~m}$ no trecho central de malha de elementos finitos (Figura 5), por um período de até 3 dias de infiltração (a depender do avanço da frente de contaminação).

O referido período de três dias foi adotado com base em referências de literatura (New Hampshire Code Of Administrative Rules, 2005, e AMORIM JÚNIOR, 2007), na qual esse intervalo foi adotado como critério de verificação de conformidade das condições de estanqueidade de bacias de tanques. Nesse enfoque, admite-se que a remoção de todo o contaminante acumulado sobre o terreno no interior da bacia de contenção deverá ser efetuada em até três dias, sendo consideradas aceitáveis as áreas em que a infiltração tenha pouco avanço nesse período.

Para a sucção matricial inicial na massa de solo, foi admitida uma variação linear da carga total com a profundidade, $h_{w}=z_{w s}-z$. Nessa formulação, $z_{w s}$ é a elevação na qual há sucção nula (nível freático) e $h_{w}$ é a carga total na elevação $z$. Foi também admitida ausência de quaisquer poluentes orgânicos no subsolo das bacias de contenção antes da ocorrência do acidente simulado. 


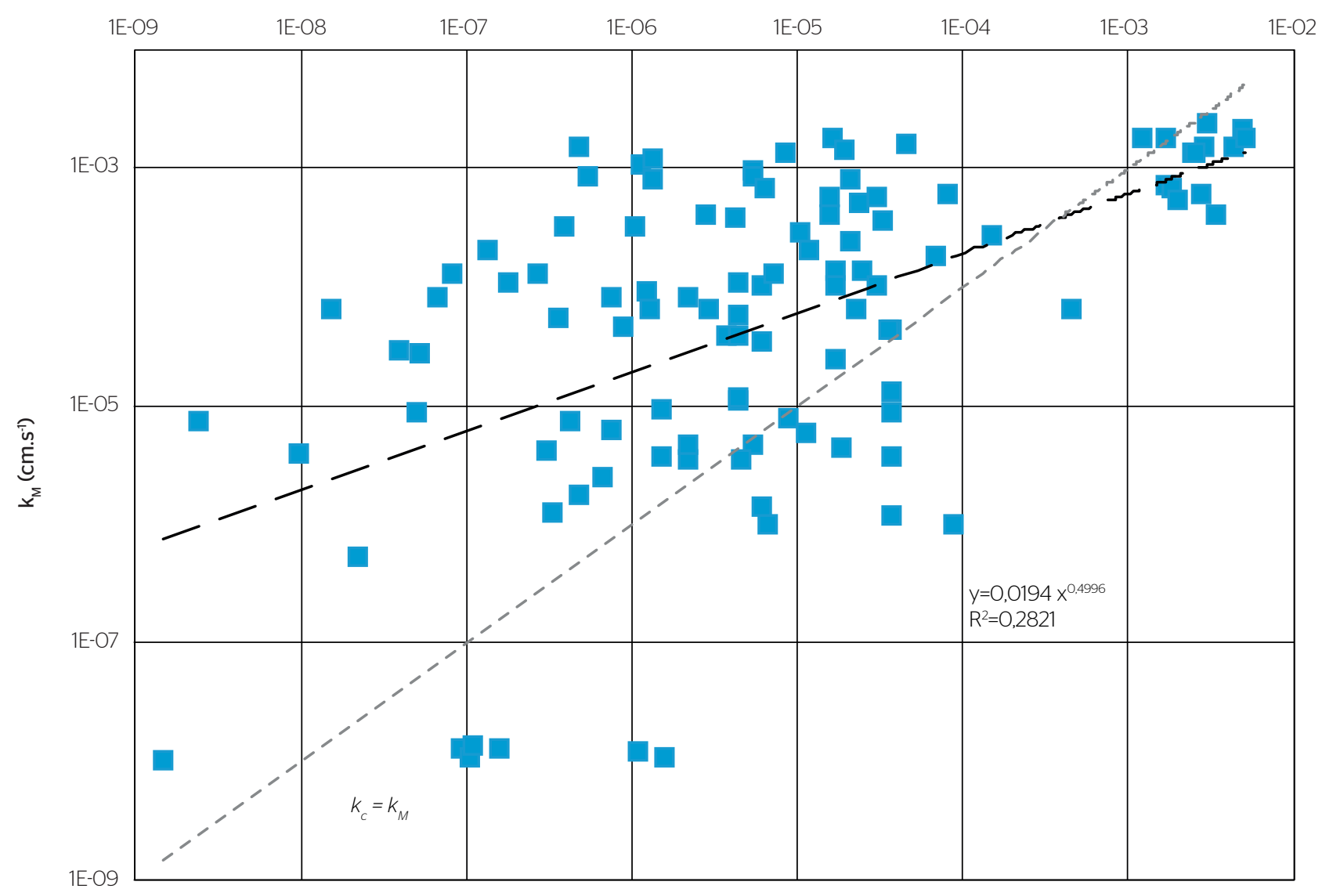

Figura 2 - Valores de condutividade medidos em laboratório $\left(K_{m}\right)$ versus condutividade calculada a partir da permeabilidade intrínseca $\left(K_{c}\right)$, para diferentes hidrocarbonetos.

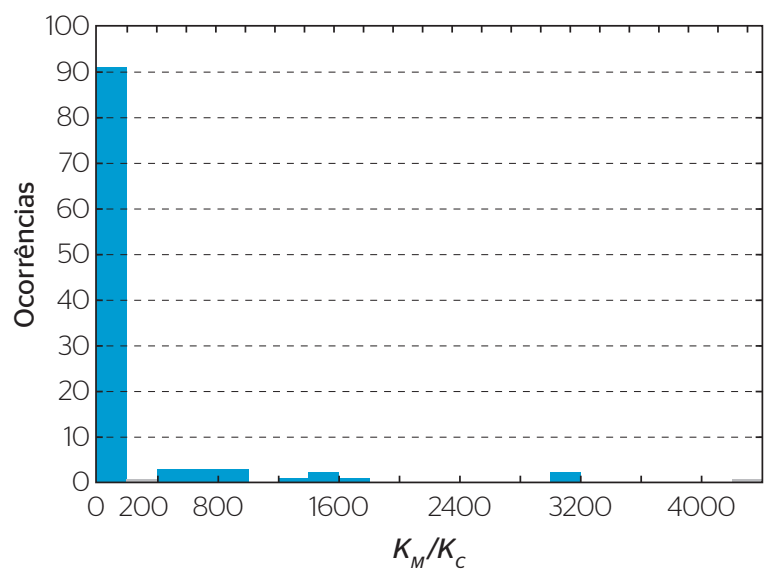

Figura 3 - Histograma dos resultados da razão $K_{M} / K_{C}$ para diferentes hidrocarbonetos.

\section{RESULTADOS}

Os resultados obtidos nas análises efetuadas para as Áreas 1 e 2, considerando-se a ocorrência de grande derramamento acidental de combustível nas bacias de contenção avaliadas, encontram-se apresentados nas Figuras 6 a 10.

As Figuras 6 a 8 exibem o avanço da frente de contaminação após hipotético acidente com vazamento de óleo diesel na Área 1.

Na Figura 6, é apresentada a previsão de contaminação do subsolo, decorridos três dias após o derramamento, considerando-se um valor de $K_{P}$ inferido a partir de $K_{W}$. Nessa figura, pode ser observada uma profundidade de contaminação bastante reduzida, condição esta que prescindiria de obras de impermeabilização no local e ratificaria o critério estabelecido na NBR 17505-2. Tal ausência de necessidade de obras de impermeabilização seria justificada por ser factível e viável a remoção do solo contaminado nessas condições, permitindo uma 


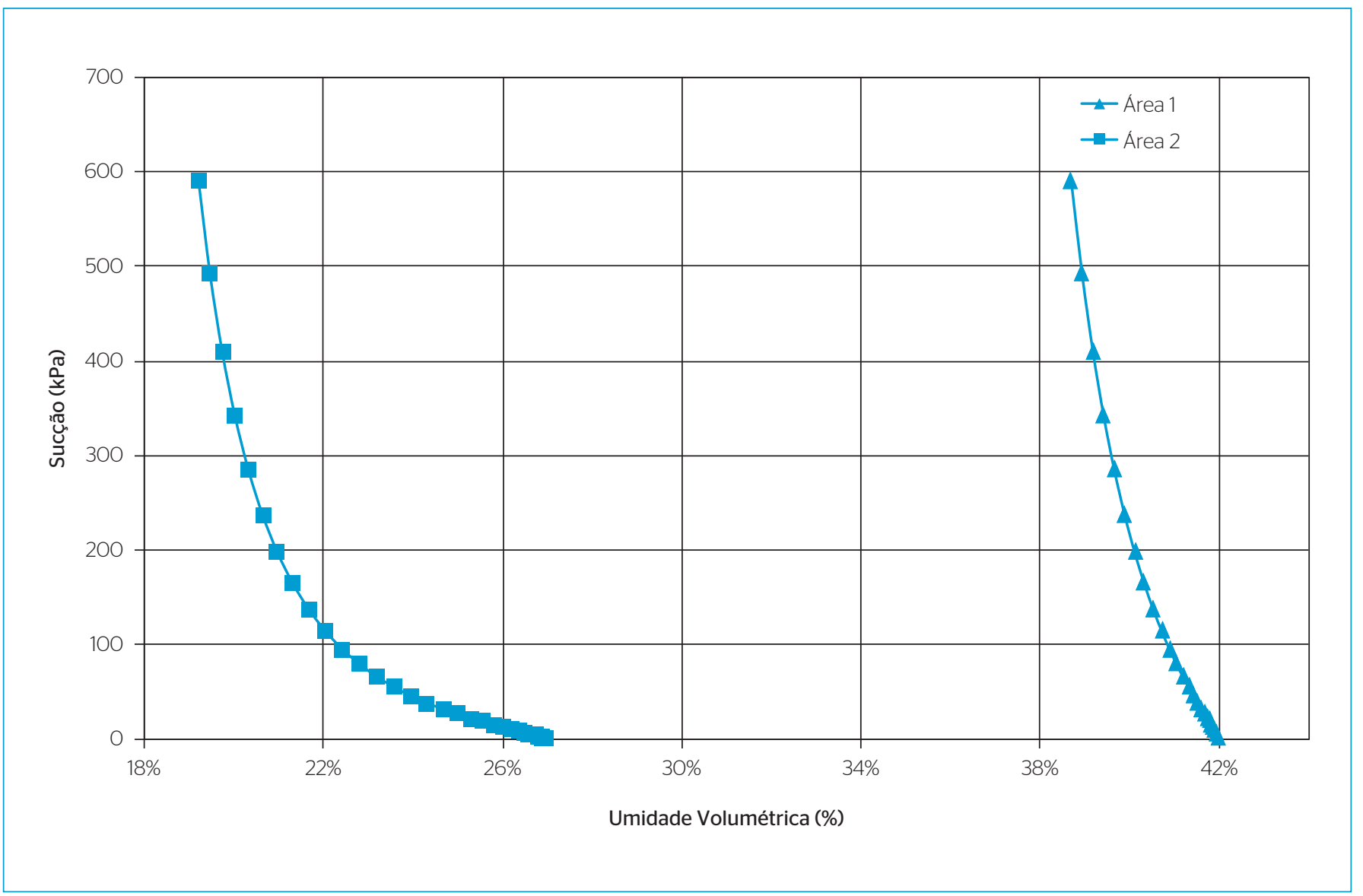

Figura 4 - Curva característica de sucção obtida para os solos das Áreas 1 e 2.

Tabela 3 - Parâmetros de análise.

\begin{tabular}{|c|c|c|c|}
\hline \multicolumn{2}{|c|}{ Área 1} & \multicolumn{2}{|c|}{ Área 2} \\
\hline$K_{S W}: 2,0 \times 10^{-7} \mathrm{~cm}^{-1}$ & $\phi .0,396$ & $K_{S W}: 6,0 \times 10^{-6} \mathrm{~cm}^{-1}$ & $\phi .0,360$ \\
\hline$S_{M}: 0,09$ & $\bar{S}_{R O}^{\max }: 0,20$ & $S_{M}: 0,04$ & $\bar{S}_{R O}^{\max }: 0,20$ \\
\hline$\alpha: 0,10 \mathrm{~m}^{-1}$ & $n: 1,17$ & $\alpha: 0,39 \mathrm{~m}^{-1}$ & $n: 1,26$ \\
\hline$\beta_{A 0}: 3,040$ & $\beta_{\text {OW }}: 1,490$ & $\beta_{A 0}: 3,040$ & $\beta_{\text {ow }}: 1,490$ \\
\hline$\eta_{R O}: 3,75$ & $\rho_{R O}: 0,832$ & $\eta_{R O}: 620$ & $\rho_{R O}: 1,024$ \\
\hline
\end{tabular}

Tabela 4 - Valores de $\mathrm{K}_{\mathrm{p}}$ adotados nas análises.

\begin{tabular}{l|c|c}
\multirow{2}{*}{ Local } & \multicolumn{2}{|c}{ Condutividade ao produto $\mathrm{K}_{\mathrm{p}}\left(\mathrm{cm}^{-1} \mathrm{~s}^{-1}\right.$} \\
\cline { 2 - 3 } & Calculada a partir de $\mathrm{K}_{\mathrm{w}}$ & Corrigida \\
\hline Área 1 (óleo diesel) & $4,5 \times 10^{-8}$ & $7,0 \times 10^{-5}$ \\
\hline Area 2 (óleo comb.) & $1,0 \times 10^{-8}$ & $4,6 \times 10^{-7}$ \\
\hline
\end{tabular}


口-

rápida reabilitação da área em estudo mesmo depois de um acidente com derrame de combustível.

As Figuras 7 e 8, contudo, contrariam tal assertiva ao exibir os resultados obtidos com $\mathrm{K}_{\mathrm{p}}$ corrigido. Em tais gráficos, podem ser observados avanços significativos e intoleráveis da frente de contaminação em períodos de tempo relativamente curtos (12 e 18 horas, respectivamente).
As Figuras 9 e 10 apresentam os resultados obtidos para a Área 2, considerando-se $\mathrm{K}_{\mathrm{p}}$ calculado e corrigido, respectivamente. Ambos os gráficos mostram um mesmo padrão de comportamento, com avanços pouco importantes da frente de contaminação. Esse comportamento, como era de ser esperado, torna a Área 2 ambientalmente adequada para conter um grande vazamento de óleo combustível por um período de até três dias.

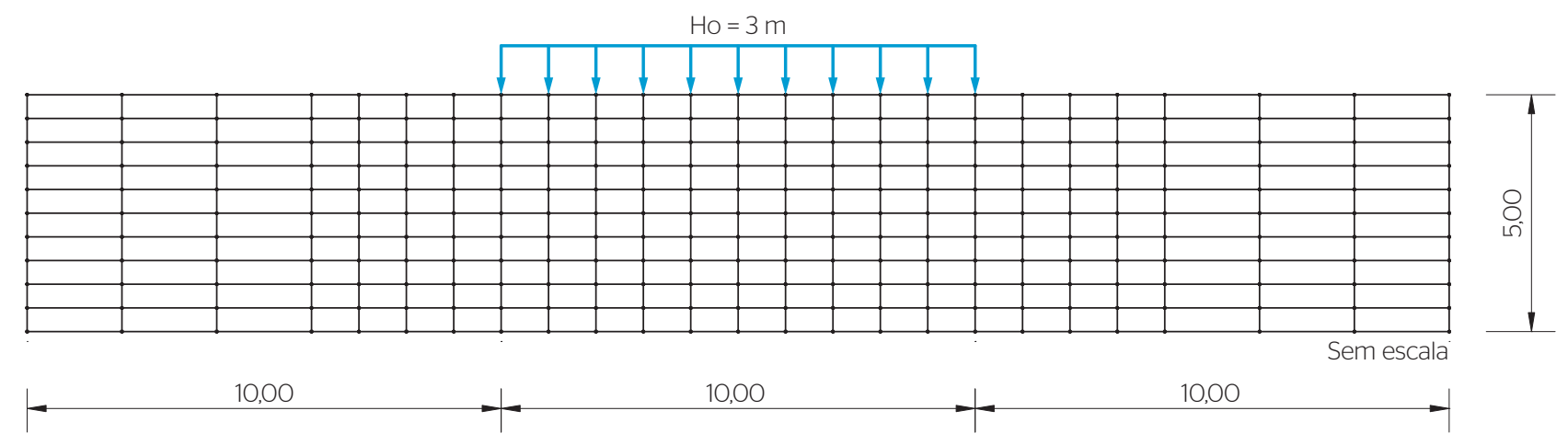

Figura 5 - Malha de elementos finitos usada nas simulações.
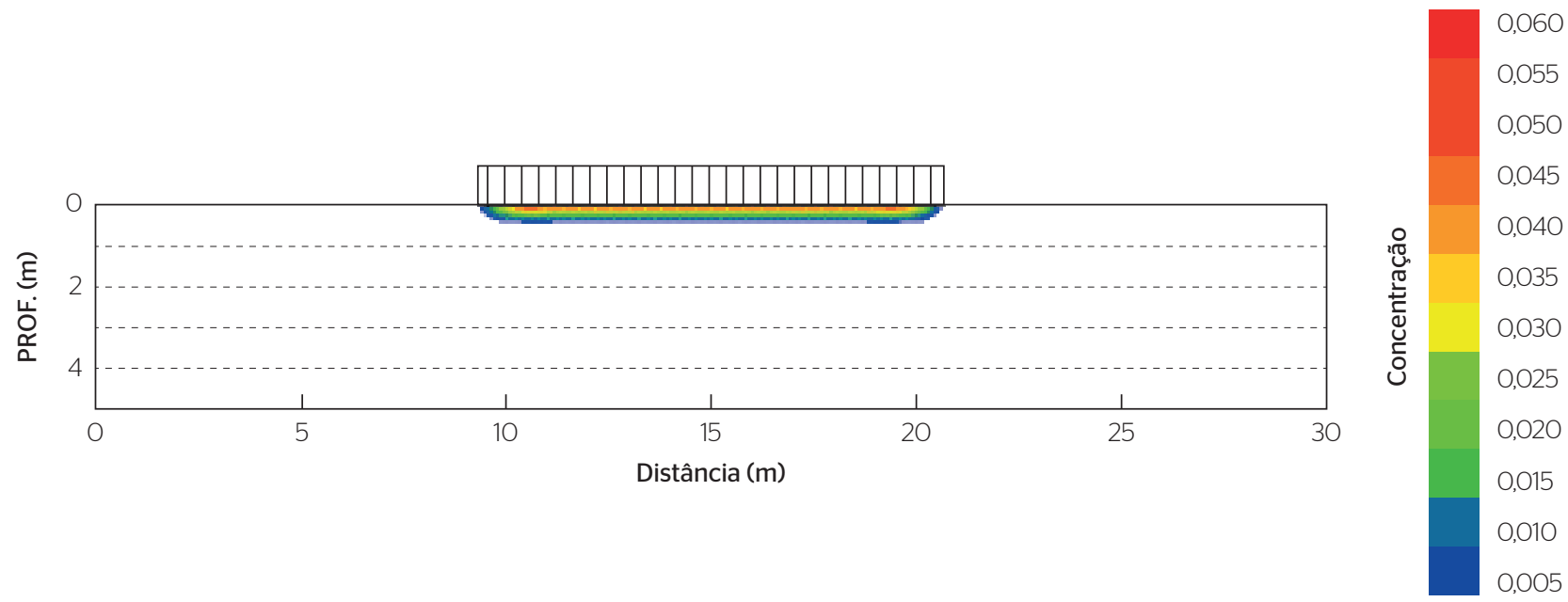

PROF:: Profundidade

Figura 6 - Saturação de óleo na Área 1 após três dias de infiltração $\left(K_{p}\right.$ calculado). 

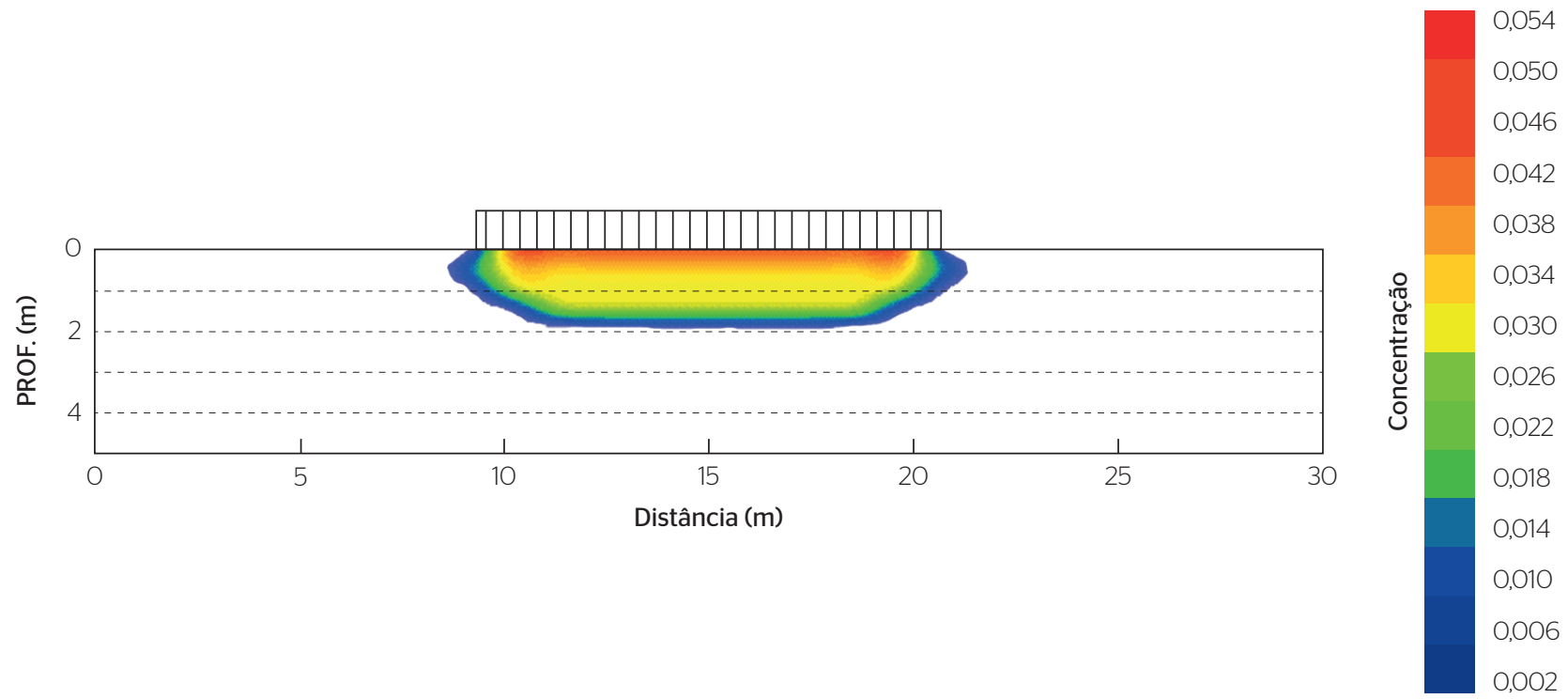

PROF.: Profundidade

Figura 7 - Saturação de óleo na Área 1 após 12 horas de infiltração $\left(K_{P}\right.$ corrigido).
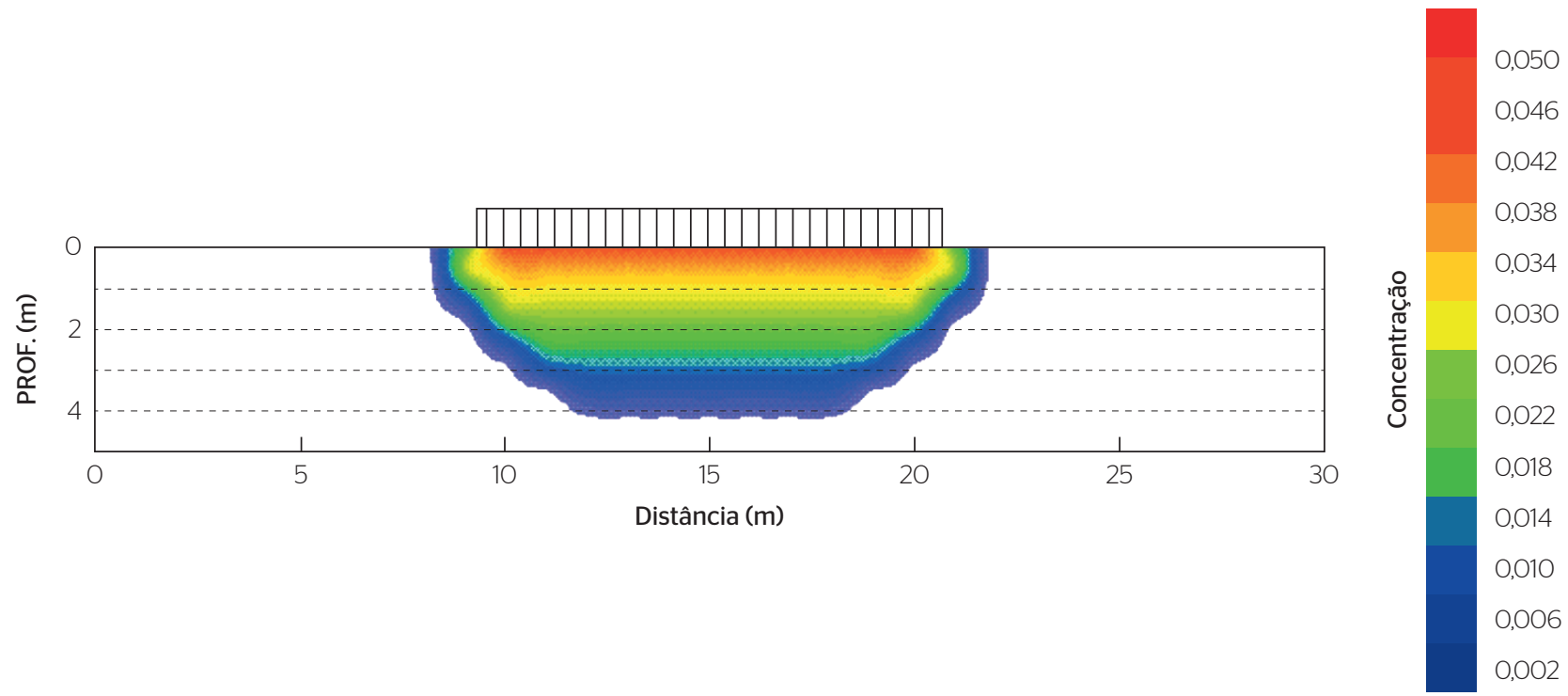

PROF.: Profundidade

Figura 8 - Saturação de óleo na Área 1 após 18 horas de infiltração ( $K_{p}$ corrigido). 

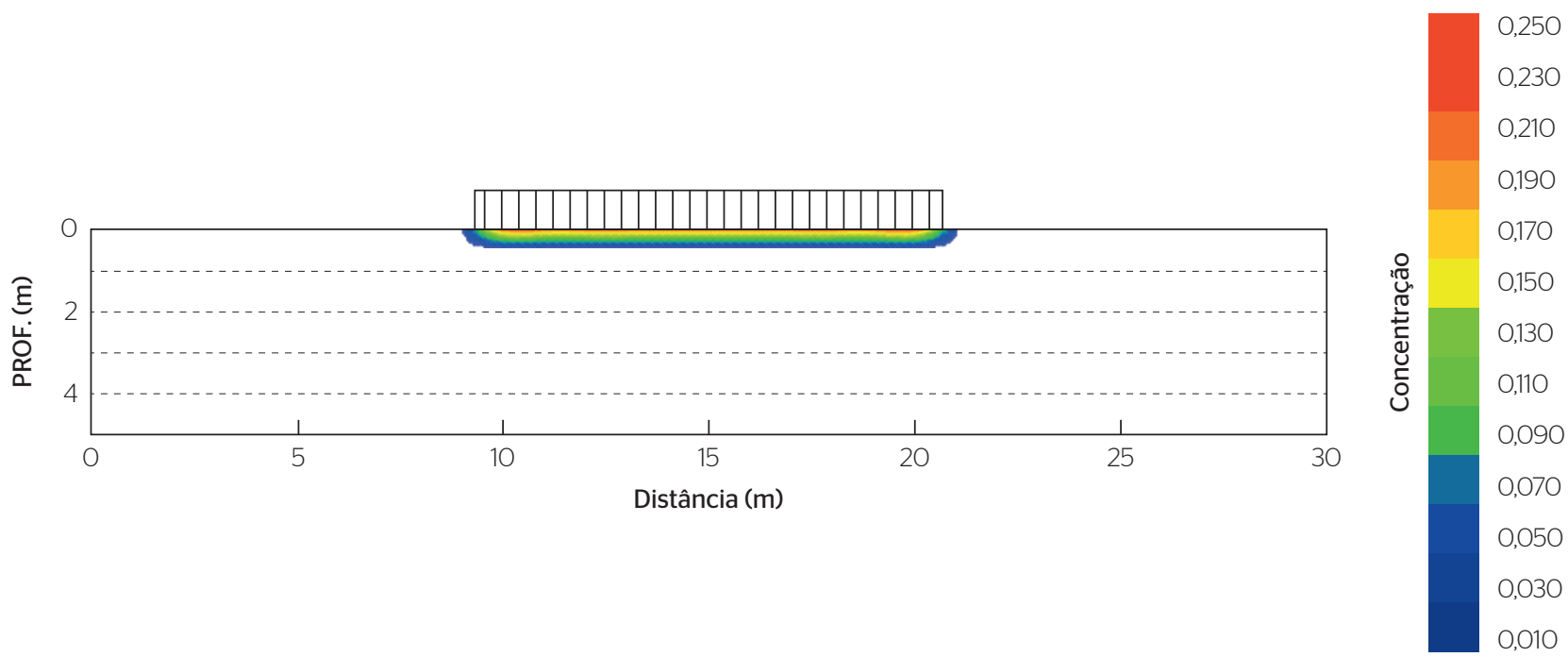

PROF: Profundidade

Figura 9 - Saturação de óleo na Área 2 após três dias de infiltração $\left(K_{p}\right.$ calculado).
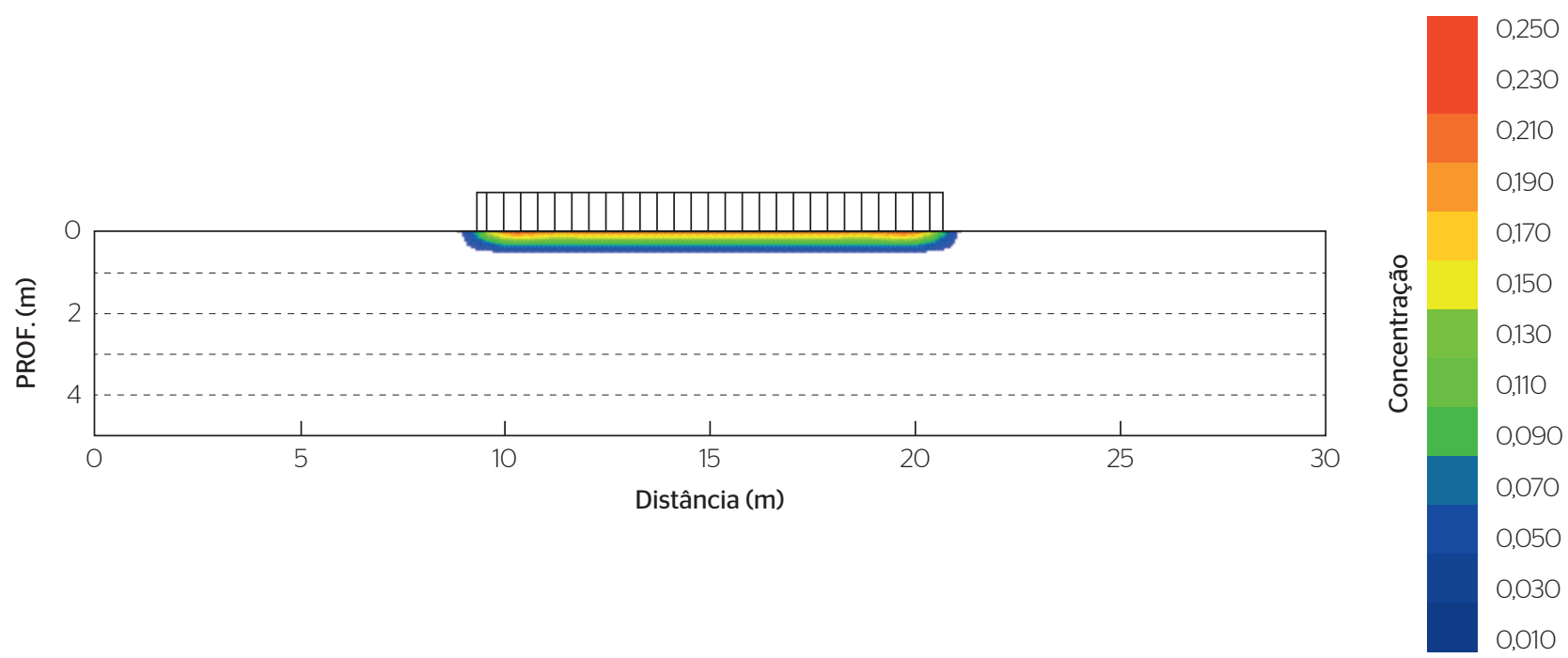

PROF: Profundidade

Figura 10 - Saturação de óleo na Área 2 após três dias de infiltração $\left(K_{p}\right.$ corrigido). 
A exemplo do observado na simulação anterior, os resultados obtidos para a Área 2 também contrariam o critério constante na NBR 17505-2. Nesse caso, ao desconsiderar as propriedades do fluido estocado, a referência normativa erroneamente aponta a necessidade de intervenção na área.

\section{CONCLUSÕES}

- Há uma carência de referências normativas nacionais adequadas a uma análise consistente das condições de estanqueidade de bacias de contenção não revestidas de tanques de armazenamento de hidrocarbonetos evidenciada pela obsolescência da NBR 17505-2 nesse quesito.

- Para o desenvolvimento de uma abordagem racional do problema, verifica-se a necessidade de se adotar critérios de verificação de estanqueidade que considerem as características e especificidades locais, tais como as propriedades de solo, o produto armazenado e as diferenças no comportamento de solos finos em contato com água e com hidrocarbonetos.

- Na ausência de recursos e tempos de resposta confiáveis a emergências, localmente determinados para cada área operacional, é prudente o uso de períodos de tempo de resposta relativamente conservadores, tais como o adotado neste trabalho (três dias) com base em referências bibliográficas. As simulações numéricas efetuadas para a Área 1 com o emprego de valores de condutividade ao óleo exibiram profundidades de infiltração inadmissíveis em intervalo de tempo consideravelmente inferior a três dias.

- Em que pese a existência de documentação normativa internacional em que são arbitradas profundidades limites para o avanço da frente de contaminação, é do entendimento dos autores que a profundidade tolerável de solo contaminado deva ser determinada individualmente para cada local, levando em consideração as características e a estratigrafia do terreno, a profundidade do NA, a presença de cursos d'água, as condições de escavação e remoção de solo contaminado, a disponibilidade de locais para destinação de resíduos, entre outros.

- A aplicação direta da regulamentação normativa em vigor no Brasil levaria, para as áreas estudadas neste trabalho, a uma inversão na avaliação das necessidades de intervenção, ao induzir a uma decisão errônea de não intervir em uma área ambientalmente vulnerável, e de acusar a necessidade de impermeabilização de uma outra área pouco susceptível à infiltração do hidrocarboneto armazenado.

\section{REFERÊNCIAS}

AMORIM JúNIOR, C.J. (2007). Avaliação dos Critérios de Impermeabilização de Bacias de Contenção da Norma ABNT NBR 17505 2/2006 para Terminais de Armazenamento de Petróleo e Derivados. 127 p. Dissertação (Mestrado em Engenharia Ambiental) - Universidade Federal de Santa Catarina, Florianópolis.

CALIFÓRNIA (EUA). (2006). California Codes. Statutes of Chapter 6.7 Health \& Safety Code - Underground Storage of Hazardous Substances. EUA, $44 \mathrm{p}$.

CORSEUIL, H.X.; MELEGARI, S.P.; OLIVEIRA, J.C.; COELHO, J.I.E. (2006). Avaliação da eficiência das bacias de contenção e do risco ambiental associado aos possíveis derramamentos de petróleo e derivados. Relatório Técnico, 115 p.

KATYAL, A.K.; KALUARACHCHI, J.J.; PARKER, C. (1991). MOFAT: A Two-dimensional Finite Element Program for Multiphase Flow and Multicomponent Transport. Center for Environmental and Hazardous Materials Studies, Virginia Polytechnic Institute and State University (EUA), $122 \mathrm{p}$.
MACHADO, S.L. (2008). Estudos geotécnicos e de Viabilidade para Adequação de Bacias de Contenção de Tanques em Terminais do Nordeste. Relatório Técnico, 292 p.

NEW HAMPSHIRE (EUA). (2005). Code of Administrative Rules. Chapter Env-Wm1400 - Petroleum Storage Facilities. Division of Waste Management, EUA, $33 \mathrm{p}$.

OLIVEIRA, J.C.S. (2001). Contaminação de Sedimentos Argilosos por Combustíveis Automotivos: Problemas de Avaliação da Permeabilidade. 127 p. Tese (Doutorado em Geologia) - Instituto de Geociências, Universidade Federal da Bahia, Salvador.

U.S. EPA. (2002). Oil Pollution Prevention and Response. NonTransportation-Related Onshore and Offshore Facilities; Final Rule. 40 CFR Part 112, EUA, $112 \mathrm{p}$

VAN GENUCHTEN, M.Th. (1980). A Closed form equation for predicting the hydraulic conductivity of unsaturated soils. Soil Science Society of America Journal, v. 44, n. 5, p. 892-898. 